\title{
Carbon Footprint as a First Step Towards LCA Usage
}

\author{
Wladmir H. Motta
}

\begin{abstract}
In order to reduce the current intensive and inefficient use of resources and especially the negative impacts on the environment, some initiatives have emerged in different areas. Life Cycle Assessment (LCA) has been one of the most accepted and used methodology. Despite this fact, there are countries where LCA is not yet fully implemented. On the other hand, there is another approach, the carbon footprint $(\mathrm{CF})$, that can follow the same life cycle approach patterns considering the phases and steps of a LCA. In this sense, this study proposes CF use as an introductory methodology of the life cycle thinking in companies at countries where LCA is still not effectively in use. The proposal is conducted through a bibliographic study and a field research. The findings point to acceptance of the proposal, considering that with the use of $\mathrm{CF}$, the companies will come to know and use the principles of life cycle thinking, thus facilitating the understanding and the implementation of LCA.
\end{abstract}

\section{Introduction}

The continued use of natural resources at rates above the planet's regenerative capacity, mainly due to production and consumption, has brought our ecosystem to a reality of unprecedented fragility. In this sense, human activities have caused negative impacts on the environment at all scales.

Among the various evidences, those related to the various parameters of the Earth system where changes are leading the Earth system away from the relative equilibrium it had known since the beginning of the Holocene can be highlighted, and there is now discussion about the use of the term Anthropocene to specify the changes in the Earth system caused by the human species in a planetary scale, taking into account the impact of the accelerated accumulation of greenhouse gases on climate and biodiversity and also the irreversible damage caused by the overconsumption of natural resources, among others [1].

A fact that reinforces this concern is the understanding that there are nine environmental boundaries, which, once overcome, can generate severe and nonlinear

\author{
W. H. Motta $(\bowtie)$ \\ CEFET-RJ, Rio de Janeiro, Brazil
}


changes on the continental and planetary scale. Some of these boundaries have already been extrapolated, such as climate change, loss of biosphere integrity, changes in the terrestrial system and changes in the biogeochemical cycles of phosphorus and nitrogen $[2,3]$.

Another alarming data was released recently by the Intergovernmental Panel on Climate Change (IPCC), where contrary to what was expected in the face of the Paris agreement, which promised a radical transformation in technologies, investments and consumption modes, new and severely worrying data from this latest study published in 2018 (Global Warming of 1.5) exposes that the huge effort to stop global warming must be carried out immediately, precisely from 2020, or the consequences will be catastrophic [4].

Faced with the challenges posed by the ecological urgency presented, some movements emerged, such as the Paris Agreement and the Sustainable Development Goals (SDG), agreements that will require innovative approaches and contributions from all, in this sense, specifically as organizations; they started to use environmental management practices, being one of the most usual ways to initiate these practices through certifications, among which is ISO 14000.

ISO 14000 deals with the need to adapt to any change in environmental conditions, and it embodies a life-cycle approach to address these environmental aspects; among the norms of this set of norms are those referring to the carbon footprint and the life cycle assessment. Among these two proposals, life cycle assessment (LCA) is considered a valuable tool in environmental sustainability for the industry, when reviewing the complex interaction between environmental aspects and the product life cycle, being today recognized as one of the main and most comprehensive environmental tools/methodologies.

However, the dissemination of the use of this methodology is not uniform in the world, and many countries still do not use it fully; on the other hand, there is the other methodology, the carbon footprint $(\mathrm{CF})$, which presents characteristics similar to LCA and brings less complexity in its implementation and may be a way to start implementing life cycle thinking in organizations.

To summarize, this chapter points out the following: (i) carbon footprint and LCA assess environmental impacts during the life cycle of products/services. The first is based on a mono-category assessment (only those related to climate change) and the second with a broader approach (multi-category based), both pointing impacts not only during the production process but also during extraction of inputs, use and end of use of products. (ii) Carbon footprint can be a first step on implementing LCA in companies. The findings point to a possibility of considering the use of the carbon footprint as a first stage in the implementation of the LCA, considering that with the use of $\mathrm{CF}$, the companies will come to know and use the principles of life cycle thinking, thus facilitating the understanding and the implementation of LCA. 


\section{Mono- and Multi-category Assessment}

In the recent past, proposals related to the reduction of environmental impacts were focused on the internal perimeter of companies, but according to current initiatives, based on the life cycle, this focus started to be supported in all phases, from the extraction of raw materials to transport, production and consumption, including final disposal and reuse. This seeks to reduce and even eliminate environmental impacts throughout the life cycle.

The life cycle assessment methodology seeks to improve the performance and environmental sustainability of production systems by providing detailed information with a view based on life cycle thinking. LCA has become a key element of environmental policies or voluntary actions in countries of the European Union, the United States, Japan, Korea, Canada, Australia and among emerging countries, such as India and, recently, China [5]. But this reality is not replicated in other countries, leaving aside, mainly developing countries.

For the United Nations Environment Program (UNEP) [6], the concept of life cycle thinking considers obtaining reliable information on environmental, social and economic impacts and makes this information available to decision-makers. It thus offers a way to incorporate sustainability into decision-making processes. It can be considered that among the various barriers related to LCA studies, the complexity of its preparation, thus consuming a lot of resources and time, is one of its main obstacles.

LCA is a multi-category methodology, as it is based on different categories of environmental impact to carry out its assessment and thus verify the necessary trade-offs, according to the options made. But in addition to this more robust and complex methodology, there are others that can be called mono-categories. This is the case for the carbon footprint that is based on only one impact category, that of greenhouse gas (GHG) emissions, related to global warming. This methodology provides reliable information on this impact, as in the case of LCA, on the life cycle.

The carbon footprint is a relatively new field of study. Its predecessor was the ecological footprint that is a measure of resource use and determines how much land area is needed to maintain a given population indefinitely [7]. The carbon footprint, however, appeared in the literature later, as described by [8], when it became more widely accepted that greenhouse gas emissions need to be reduced to avoid overheating the planet. Carbon footprint (CF) has quickly become a widely accepted term to further stimulate consumers' growing concern about issues related to climate change, being the instrument used to describe GHG emissions [9]. 


\subsection{Standards Related to $L C A$ and $C F$}

Among the standards, ISO 14000 standard was initially developed with proposals for standards that organizations would follow to minimize the harmful effects on the environment generated by their activities [10]. Like ISO 9000, ISO 14000 also provides practical implementation of criteria, which includes plans aimed at making decisions that favour the prevention or mitigation of environmental impacts. The standard of management of the system in families of norms establishes requirements to direct the organization of processes that influence quality (ISO 9000) or processes that influence the impact of the organization's activities on the environment (ISO 14000).

ISO 14000 represents a voluntary international environmental standard that focuses on the structure, implementation and maintenance of an environmental management system in order to motivate organizations to systematically address the environmental impacts of their activities and establish a common approach to the challenges imposed by the ecological urgency experienced [10].

ISO 14001 standard establishes the organization's environmental management system and thus [10]:

- Promotes the assessment of the environmental consequences of the organization's activities

- Seeks to meet society's demand

- Determines policies and objectives based on the environmental indicators defined by the organization (they can portray needs from the reduction of pollutant emissions to the rational use of natural resources)

- Results in cost reduction, service provision and prevention

- Is applied to activities that may affect or affect the environment

- Is applicable to the organization as a whole

The ISO 14040 series of standards describes the principles and structure of a life cycle assessment [11]; in this sense, ISO 14044 specifies requirements and provides guidelines for LCA. As pointed out by [12], these standards include the definition of the purpose and scope of the LCA, the life cycle inventory analysis (LCI) phase, the life cycle impact assessment phase, the life cycle interpretation phase, communication and critical review of the LCA, the limitations of the LCA, the relationship between the phases of the LCA and considerations for using value choices and optional elements.

In reference to the carbon footprint, the first standard that defined it was the Green House Gas Protocol (GHG Protocol) [13], an initiative that originated in 1998, which brings together members of academia, governments and NGOs, under the coordination of the World Business Council for Sustainable Development (WBCSD) and the World Resources Institute (WRI).

The GHG Protocol formed the basis for most other carbon footprint standards. There are currently three highlighted standards for calculating the carbon footprint: ISO 14067:2018; GHG Protocol Product Life Cycle Accounting and Reporting 
Standard (World Resources Institute and the World Business Council for Sustainable Development); and PAS 2050:2011 specification for the assessment of the life cycle greenhouse gas emissions of goods and services, developed by the British Standards Institution (BSI).

As for the carbon footprint normalized by ISO, in addition to ISO 14067, there are two other standards that were initially presented in 2006, namely, ISO 14064 and ISO 14065. ISO 14064, management of GHG emissions and removals, establishes standards for the quantification, monitoring and verification/validation of GHG emissions, while ISO 14065 addresses the requirements for GHG project validation and verification organizations [14]. ISO 14067: 2018 was based on the current ISO standards related to life cycle assessments (ISO 14040, ISO 14041, ISO 14042, ISO 14043 and ISO 14044) for the details for quantification, on standards related to environmental labels and statements (ISO 14020, ISO 14024 and ISO 14025 ) for the formatting for communication, specifies principles, and on requirements and guidelines for the quantification and communication of a product's carbon footprint [18]. This being the closest standard to ISO standards related to LCA.

For a world that continues to face this ecological urgency, organizations must continue/start to recognize the need to manage their environmental challenges and contribute to finding solutions to this common problem. Thus, the use of organizations of methodologies such as CF and LCA is very important in the face of this enormous challenge.

\section{Methods and Data}

This theoretical chapter aims at investigating the relationship between LCA and $\mathrm{CF}$. Based on input from the literature on LCA and CF, the available evidence for this relationship was analyzed in the context of using CF as a predecessor to LCA implementation as a first step towards effective application introducing life cycle thinking. To structure the debate, a conceptual approach was carried out, and a field research on international researchers' and practitioners' perceptions on the potentially of the proposal to have CF as a first step to LCA usage will be presented.

\subsection{Illustrative Case: Testimony of Experts}

To add to the debate on the potentially positive use of the $\mathrm{CF}$ as a predecessor of the LCA, an illustrative case on international researchers' and practitioners' perceptions on this proposal will be presented. 


\subsubsection{Data Collection and Sample}

Data collection is aimed at identifying the following aspects (among others): the state of the art of the LCA and the relationship between CF and LCA. For this purpose, a survey was designed which was disclosed and submitted through the LC Net, November-December 2015 edition, the newsletter of the Life Cycle Initiative. SurveyMonkey was used - an online survey development cloud-based software, which provides customizable surveys - for the data collection via web. The survey consisted of 15 questions organized in 9 categories according to the aspects being investigated. For the purposes of this chapter, though, it will discuss only the data related to the relationship between $\mathrm{CF}$ and LCA, one of the categories presented at this survey.

The questions covering this topic were structured as open questions and are composed of two questions that sought to understand at what stage is the use of the carbon footprint and validate the proposal that this can be a tool to promote the use and dissemination of LCA.

The Life Cycle Initiative was chosen to be the channel to access international researchers and practitioners with experience on LCA as it is regarded as a worldwide influential organization on the issues concerning LCA practices and its dissemination. At the time of data collection, November-December 2015, 106 Life Cycle Initiative members participated on the survey. The number of international respondents and the scope of their place of work/origin in 31 countries expressed a higher frequency of European countries with $67.0 \%$, followed by North America with $16.0 \%$. Regarding the time of experience, the verified distribution demonstrated a maturity of the researchers/professionals who participated, since $66.0 \%$ of the respondents had more than 6 years of experience with LCA.

\subsection{Survey Responses}

There were two questions on the questionnaire considering this topic. The first asked about the use and the way of using the carbon footprint in countries, seeking to understand if the methodology was already effectively used and if it would be a feasible option and already used as a first stage before the LCA. 99 responses were received: $65(65.7 \%)$ were positive regarding the widely use of the carbon footprint, $4(4.0 \%)$ did not know how to position themselves and $30(30.3 \%)$ were negative concerning its use (e.g. Fig. 1). Of the 31 countries whose specialists participated in the survey, only 2 did not use the carbon footprint effectively.

The second research question was related to the proposal to use the carbon footprint as a facilitator and first step towards the dissemination of LCA practice. 97 responses were received: $71(73.2 \%)$ were positive; $15(15.5 \%)$ had restrictions on the LCA being more complete and requiring more details in its execution, in addition to presenting restrictions on the use of the carbon footprint as a decision tool; 2 
Fig. 1 Usage of carbon footprint in countries

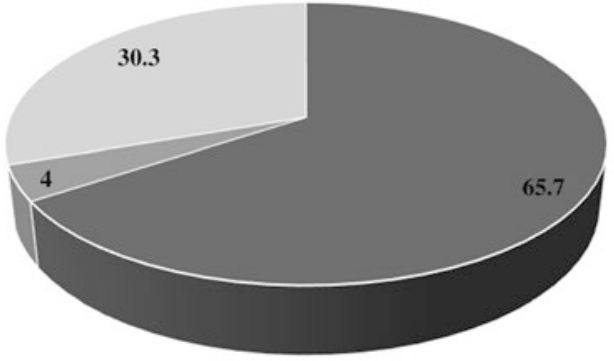

- widely use of CF = there is no widely use of CF " did not position themselves

Fig. 2 Use of the carbon footprint as a first step towards the dissemination of LCA practice

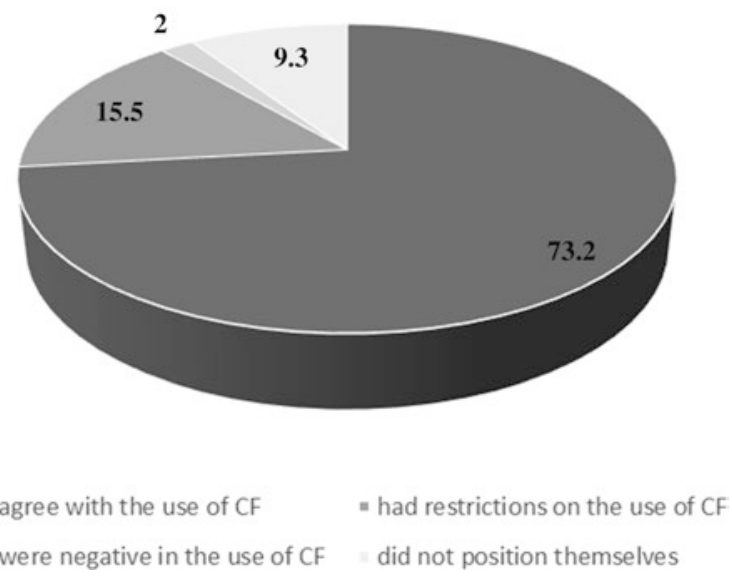

$(2.0 \%)$ were negative regarding its use as a first step in implementing an LCA; and $9(9.3 \%)$ were unable to position themselves (e.g. Fig. 2).

The comments received on this proposal to use the carbon footprint as a first step towards the effective implementation of LCA were divided into three groups. One group presented positive comments on the proposals, consisting of 15 placements; another group with 9 placements presented what could be improved after the execution of the carbon footprint. The third and last group, with 14 comments, criticized the use of the carbon footprint as a precursor to LCA.

A compilation of the positive comments regarding the use of $\mathrm{CF}$ as a precursor to the LCA is that when conducting a carbon footprint assessment, companies come to better understand direct and indirect emissions; they come to better understand what is the approach of the life cycle and the fundamental stages of an LCA study and recognize the needs of people and resources, in addition to becoming aware of the interpretation of the results when making decisions. In this way, carbon footprint requires the execution of the most difficult parts of an LCA study, and to complement this initial study, it would be necessary to basically only collect additional data 
(the multi-criteria aspect) on the processes already verified in the calculation of the carbon footprint. This evolution towards an LCA study would be relatively simple.

In relation to the comments that indicated an acceptance but with a clear understanding of the differences and the needs of future actions, we have as a compilation that for companies, it is easier to start with the carbon footprint to understand the concept of LCA. The company may be frightened when faced with many categories of impact that at first may not be relevant to its products. The use of a single criterion can help for simplicity, but it involves a lot of uncertainty and choices based on a single factor. The interpretation of an LCA study is more technical due to the different impact categories addressed and is also more complex than that of the data generated by a $\mathrm{CF}$.

As for the negative comments, the compilation of these positions points to a concern that a complete LCA study is more complex than just an accounting of GHG gases, made by a CF. The use of the carbon footprint may limit the understanding and scope of environmental issues in companies, making matters that are extremely complex really simplistic. Companies that perform a $\mathrm{CF}$ may not fully understand the concept of the life cycle and may be satisfied with just this study without understanding that they can do more through an LCA study. In the survey, $81.82 \%$ of respondents reported that the tool is used in their countries of residence/ professional practice, a scope that covers 28 of the 30 countries involved in the research. The proposal to use the carbon footprint as a precursor to the LCA was accepted by the community of researchers/international experts with an approval of $73.20 \%$ of the respondents and a perceived concern on the part of $15.46 \%$ of the respondents regarding a possible loss of perception of the advantages of using the LCA methodology.

\section{Discussion}

In the survey, $81.82 \%$ of respondents reported that the tool is used in their countries of residence/professional practice, a scope that covers 28 of the 30 countries involved in the research. The proposal to use the carbon footprint as a precursor to the LCA was accepted by the community of researchers/international experts with an approval of $73.20 \%$ of the respondents, with a perceived concern on the part of $15.46 \%$ of the respondents regarding a possible loss of perception of the advantages of using the LCA methodology.

This concern is due to the fact that because the carbon footprint is mono-category, it verifies the impacts related only to its category (GHG emissions/global warming) and provides unilateral decision-making aimed at reducing the environmental impacts related to this category and that may eventually promote other impacts not perceived by the tool (since they are not evaluated by the tool). This fact does not occur with the LCA methodology, since it measures the impacts related to a considerable group of different categories and is able to provide information on the 
trade-offs that will occur due to the decisions taken with reference to these evaluated categories.

As a result of these respondents' cautious positions and positive opinions regarding the proposal, 38 comments were analysed, and from these it can be concluded that according to what was reported in the survey, the carbon footprint, although simpler than the LCA, brings the life cycle approach, its methodology and its steps into companies and can collaborate as their first contact with this approach model; the carbon footprint provides insight into the impacts generated and their dimensions for companies; the use of the carbon footprint becomes a facilitator as the life cycle study is carried out for only one impact category.

As negative aspects pointed out, several of them are relevant and are presented here: there is a need for other knowledge besides those related to the impacts responsible for global warming to be acquired and present when carrying out the LCA study; the possible difficulty in conducting the interpretation of the LCA study when carried out by the company that initially only conducted carbon footprint studies, due to the trade-offs visualized and glimpsed in as a result of the LCA studies; the fear that the methodology used to execute the carbon footprint is based on the GHG protocol or PAS 2050, which could distance the company from understanding the life cycle approach and the use of the LCA methodology; concern was shown for small businesses that would not be able to afford the costs of an LCA study; limitations regarding the need to use software for LCA studies when, for carbon footprint studies, they are not necessary; and concerns about the possibility that after the use of the carbon footprint the use of the LCA may be disowned.

The carbon footprint, being considered an integral part of an LCA study, follows the same pattern (when based on ISO 14067) of the life cycle approach as the phases and steps to be followed in its application, thus bringing the practice of the life cycle approach to the companies that execute it. Another issue regarding the use of the carbon footprint as a first step in the implementation of the LCA is that this methodology, mainly due to the results and commitments assumed by the countries participating in COP 21, tends to have greater use and eventual collection, even legal, in these countries.

\section{Conclusion}

The carbon footprint, being considered an integral part of an LCA study, follows the same pattern (when based on ISO 14067) of the life cycle approach as the phases and steps to be followed in its application, thus bringing the practice of the life cycle approach to the companies that execute it. Another issue regarding the use of the carbon footprint as a first step in the implementation of the LCA is that the CF methodology, mainly due to the commitments assumed by the countries participating in the COP 21, Paris Agreement, tends to have greater interest and use in the countries signatories to the agreement. 
The use of the carbon footprint also directly corroborates other objectives to be achieved by nations, referring here to the Sustainable Development Goals (SDGs). Among the 17 objectives assumed, the carbon footprint has a direct relationship, especially with the thirteenth objective - "Take urgent measures to combat climate change and its impacts", in addition to having other interfaces with others of the 17 objectives.

The concern reported in the survey by a portion of the respondents, regarding a possible replacement of the LCA by the carbon footprint, should be considered, but the purpose of this study is not to propose CF use as the main methodology, but to enable companies to have contact and experience with the life cycle approach, and from this first experience, they can evolve to the admittedly more complete methodology which is the LCA.

Thus, the present study suggests that the carbon footprint should be considered as a methodology to be used as a precursor to LCA studies in companies, a factor that tends to facilitate a comprehensive implementation of LCA in countries where this practice is not yet a reality. It is hoped that this study can motivate more indepth research and practical applications that can reinforce the pointed interrelation and proposal.

\section{References}

1. Issberner, L.-R., \& Lená, P. (2018). Anthropocene: The vital challenges of a scientific debate. In The UNESCO Currier, Abril/June.

2. Rockstrom, J., \& Steffen, W. (2009). Planetary boundaries: Exploring the safe operating space for humanity. Ecology and Society, 14(2), 32.

3. European Commission EUROPE 2020: A Strategy for Smart, Sustainable and Inclusive Growth, Brussels, 3.3.2010. Communication from the Commission, COM (2010/ 2020), 2010.

4. IPCC. (2018). Global Warming of $1.5^{\circ} \mathrm{C}$., in: https://www.ipcc.ch/sr15/.

5. Guinée, J. B., Heijungs, R., \& Huppes, G. (2011). Life cycle assessment: Past, present and future. Environmental Science \& Technology, 45(1), 90-96.

6. United Nations Environment Programme - UNEP/SETAC, Greening the economy through life cycle thinking: Ten years of the UNEP/SETAC Life Cycle Initiative, Paris, 2012.

7. Wackernagel, M., \& Rees, W. E. (1996). Our ecological footprint reducing human impact on the Earth. New Society Publishers.

8. Wiedmann, T., \& Minx, J. (2008). Chapter 1: A definition of 'Carbon Footprint'. In C. C. Pertsova (Ed.), Ecological economics research trends (pp. 1-11). Nova Science Publishers.

9. Esty, D. C., \& Winston, A. S. (2008). O verde que vale ouro. Elsevier.

10. ISO. ISO 14001:2015 Environmental management systems - Requirements with guidance for use, 2015.

11. ISO. ISO 14040, Environmental management - Life cycle assessment - Principles and framework. Geneva, Switzerland, 2006.

12. Palma-Rojas, S., Paiva-Castro, P., Gama-Lusta, C., \& Lamb, C. R. (2012). Sistema brasileiro de inventário de ciclo de vida (SICV Brasil) e a ISO 14.044:2009. In Congresso Brasileiro em Gestão do Ciclo de Vida de Produtos e Serviços, 3., Maringá.

13. World Resources Institute and World Business Council For Sustainable Development, The greenhouse gas protocol, Technical Report, 2000.

14. ISO. ISO14067, Carbon footprint of products - Requirements and guideline, 2018. 
Open Access This chapter is licensed under the terms of the Creative Commons Attribution 4.0 International License (http://creativecommons.org/licenses/by/4.0/), which permits use, sharing, adaptation, distribution and reproduction in any medium or format, as long as you give appropriate credit to the original author(s) and the source, provide a link to the Creative Commons license and indicate if changes were made.

The images or other third party material in this chapter are included in the chapter's Creative Commons license, unless indicated otherwise in a credit line to the material. If material is not included in the chapter's Creative Commons license and your intended use is not permitted by statutory regulation or exceeds the permitted use, you will need to obtain permission directly from the copyright holder.

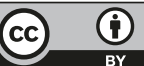

